\title{
miR-9-3p inhibits glioma cell proliferation and apoptosis by directly targeting FOXG1
}

\author{
JIANWEN ZHEN, HENGXUN ZHANG, HONGZHI DONG and XIAOPENG TONG \\ Department of Cardio-cerebrovascular Diseases, The Affiliated Hospital of Xizang Minzu University, \\ Xianyang, Shaanxi 712082, P.R. China
}

Received June 3, 2019; Accepted February 11, 2020

DOI: $10.3892 / \mathrm{ol} .2020 .11725$

\begin{abstract}
There is accumulating evidence indicating that microRNA (miR)-9-3p expression is abnormal in patients with glioma; however, the role of miR-9-3p in glioma remains unclear. In the present study, reverse transcription-quantitative PCR and immunohistochemical assays were conducted to assess miR-9-3p and forkhead box G1 (FOXG1) expression, respectively. A luciferase reporter assay was performed to confirm the target of miR-9-3p. Moreover, cell counting kit-8 and flow cytometry assays were used to assess proliferation and apoptosis, respectively. The present study demonstrated that miR-9-3p is significantly downregulated, and FOXG1 is significantly upregulated, in patients with glioma. miR-9-3p overexpression inhibited proliferation and increased the apoptosis of both U87MG and TG-905 cells. In addition, FOXG1 was identified as a direct target of miR-9-3p, and FOXG1 silencing enhanced the inhibitory effect of miR-9-3p on proliferation and apoptosis in U87 MG and TG-905 cells. In conclusion, the present results suggest that miR-9-3p may suppress malignant biological properties by targeting FOXG1. Thus, miR-9-3p may serve as a diagnostic target and novel prognostic marker in patients with glioma.
\end{abstract}

\section{Introduction}

Gliomas are the most frequently occurring type of brain tumor in adults, accounting for 45-55\% of primary nervous system tumors, worldwide (1). They are characterized by high aggressiveness, due to local diffuse infiltration, and a poor prognosis $(2,3)$. High-grade gliomas, which are classified as grade III or IV by the World Health Organization (WHO) classification system (4), are the brain tumor type with the highest mortality rate, and exhibit heterogeneity at both the microscopic and molecular levels $(5,6)$.

Correspondence to: Dr Xiaopeng Tong, Department of Cardiocerebrovascular Diseases, The Affiliated Hospital of Xizang Minzu University, 6 Wenhui East Road, Xianyang, Shaanxi 712082, P.R. China

E-mail: txpbiology@163.com

Key words: glioma, microRNA-9-3p, forkhead box G1, proliferation, apoptosis, U87 cells, TG-905 cells
Despite recent advancements in the treatment of glioma, the median survival time of patients is $<16$ months (7). Therefore, a comprehensive understanding of the mechanisms underpinning gliomagenesis is imperative, and may provide new insights into the clinical management of glioma.

microRNAs (miRNAs) are short non-coding RNA molecules that serve crucial roles in the regulation of numerous fundamental cellular processes by binding to the 3'-untranslated regions of target gene (8). Multiple studies have indicated that various miRNAs are aberrantly expressed in glioma tissues: Xiong et al (9) identified that miR-320a inhibits the invasion and migration of glioma cells via the targeting of aquaporin 4. Furthermore, Liu et al (10) demonstrated that miR-93 serves as an oncogene in glioma by directly targeting retinoblastoma-like protein 2 . Previous studies have also reported that miR-9 can influence the angiogenesis and apoptosis of glioma by regulating MYC (11) and homocysteine inducible ER protein with ubiquitin like domain 1 (12). However, the mechanism behind miR-9 regulation of glioma cell proliferation and apoptosis is yet to be elucidated.

Forkhead box G1 (FOXG1), also known as brain factor 1, is an important member of the forkhead box transcription factor family. FOXG1 is upregulated in a variety of malignant tumors and is involved in multiple developmental pathways in tumor cells, including proliferation, differentiation, cell cycle regulation and apoptosis (13). Silencing or down-regulation of FOXG1 inhibits the invasion and metastasis of colorectal cancer cells (14), and also can inhibit the proliferation of glioma cells (15). Furthermore, Shibata et al (16) revealed that miR-9 regulates Cajal-Retzius cell differentiation by targeting FOXG1 in the mouse medial pallium. However, to the best of our knowledge, whether miR-9 can inhibit the proliferation and apoptosis of glioma via the targeting of FOXG1 is yet to be determined.

The present study aimed to investigate the association between FOXG1 and miR-9-5p. It was identified that miR-9-5p is expressed at a lower level in glioma tissues compared with adjacent paracancerous tissues. In addition, FOXG1 was identified as a direct target of miR-9-5p that mediates glioma cell proliferation and apoptosis.

\section{Materials and methods}

Tissue samples. All clinical samples were obtained from the Affiliated Hospital of Xizang Minzu University (Xianyang, 
China) between May 2018 and February 2019. Glioma tissues from 5 patients (age range, 24-60 years; sex, 3 males and 2 females) and glioma-adjacent tissues from 5 individuals (age range, 23-57 years; sex, 2 males and 3 females) were fixed with $4 \%$ paraformaldehyde for $48-72 \mathrm{~h}$ at room temperature and embedded in paraffin. For all patients, the original diagnosis and tumor grading were conducted in a blinded manner by two experienced pathologists, according to the principles defined by the WHO classification system. Written informed consent was obtained from all patients, prior to surgery. The present study was approved by the Ethics Review Board of the Affiliated Hospital of Xizang Minzu University.

Cell culture and transfection. The human glioblastoma U87 MG (CL-0238) and TG-905 (CL-0309) cell lines were purchased from Procell Life Science \& Technology Co., Ltd. (the origin of glioblastoma U87 MG cell is unknown and the cell line is preserved at the ATCC). U87 MG cells were cultured in MEM (Hyclone; Logan) supplemented with 10\% FBS (Gibco; Thermo Fisher Scientific, Inc.) and 1\% penicillin and streptomycin (Beijing Solarbio Science \& Technology Co., Ltd.), in a humidified atmosphere of $5 \% \mathrm{CO}_{2}$ at $37^{\circ} \mathrm{C}$. TG-905 cells were cultured in RPMI-1640 medium supplemented with $10 \%$ FBS and $1 \%$ penicillin and streptomycin. For sub-culturing purpose, cells were incubated with $0.25 \%$ trypsin at $37^{\circ} \mathrm{C}$ and cultures were harvested at $80 \%$ confluency.

Small interfering (si)RNA against FOXG1 (FOXG1-siRNA), negative control siRNA (NC-siRNA), and the miR-9-3p mimic and negative control molecules ( $\mathrm{NC}$ mimic) were purchased from Guangzhou RiboBio Co., Ltd. Cell transfection was performed using riboFECT ${ }^{\mathrm{TM}} \mathrm{CP}$ Transfection kit (Guangzhou RiboBio Co., Ltd.), according to the manufacturer's protocol, with $50 \mathrm{pmol} / \mathrm{ml} \mathrm{miR-9-3p} \mathrm{mimic} \mathrm{and} \mathrm{NC}$ mimic, and $40 \mathrm{pmol} / \mathrm{ml}$ FOXG1-siRNA and NC-siRNA. Cells were seeded into 6-well plates at a density of $1 \times 10^{5}$ cells/well until they reached $50 \%$ confluence, $24 \mathrm{~h}$ before transfection. Fresh medium was replaced after $6 \mathrm{~h}$. Following transfection for $24-48 \mathrm{~h}$ at $37^{\circ} \mathrm{C}$, cells were collected for RT-qPCR or western blotting analyses. The FOXG1-siRNA and NC-siRNA sequences were as follows: FOXG1-siRNA forward, 5'-CGU UUUACACACAUUUGCATT-3' and reverse, 5'-UGCAAA UGUGUGUAAAACGTT-3'; NC-siRNA forward, 5'-UUC UCCGAACGUGUCACGUTT-3' and reverse, 5'-ACGUGA CACGUUCGGAGAATT-3'.

Immunohistochemistry. The paraffin-embedded glioma and glioma-adjacent tissues were cut into $4 \mu \mathrm{m}$ thick slices, deparaffinized using xylene and rehydrated with graded alcohol solutions (100\% alcohol I for $3 \mathrm{~min}, 100 \%$ alcohol II for $3 \mathrm{~min}$, 95\% alcohol for $3 \mathrm{~min}, 85 \%$ alcohol for $3 \mathrm{~min}$ and $75 \%$ alcohol for $3 \mathrm{~min}$, respectively). Subsequently, antigen retrieval was performed using $3 \%$ hydrogen peroxide at room temperature for $15 \mathrm{~min}$. Cells were then blocked with $5 \%$ bovine serum albumin (BSA; Beyotime Institute of Biotechnology) at room temperature for $20 \mathrm{~min}$. The sections were incubated with primary antibody FOXG1 (1:500; cat. no. ab18987; Abcam) at $4^{\circ} \mathrm{C}$ overnight, warmed for $30 \mathrm{~min}$ in a $37^{\circ} \mathrm{C}$ incubator, and then incubated with goat anti-rabbit biotinylated secondary antibodies (1:100; cat. no. BA1003; Wuhan Boster Biological Technology, Ltd) for $30 \mathrm{~min}$ at $37^{\circ} \mathrm{C}$. The sections were then stained with Strept Avidin Biotin Enzyme Complex (Wuhan Boster Biological Technology, Ltd.) for $30 \mathrm{~min}$ at $37^{\circ} \mathrm{C}$, and then counterstained with hematoxylin for $2 \mathrm{~min}$ at room temperature, before being treated with 3,3'-diaminobenzidine, which was used as the chromogen, for $5 \mathrm{~min}$ at room temperature. In the negative control group, PBS was used in place of the primary antibody. Five visual fields were randomly selected and assessed for immunoreactive using a light microscope (Nikon Corporation; magnification $\mathrm{x} 400$ ). The images were analyzed using Image-Pro Plus software (version 6.0; Media Cybernetics, Inc.).

Western blotting. $48 \mathrm{~h}$ after transfection, harvested cells were washed with PBS and lysed using radioimmunoprecipitation assay lysis buffer (Wuhan Boster Biological Technology, Ltd). Total protein was quantified using the bicinchoninic acid Protein Assay kit (Wuhan Boster Biological Technology, Ltd.). Equal amount of protein ( $20 \mu \mathrm{g} / \mathrm{lane})$ were separated using SDS-PAGE on a $10 \%$ gel and then transferred onto a polyvinylidene fluoride membrane (EMD Millipore). Subsequently, the membranes were blocked for $1 \mathrm{~h}$ at room temperature using $5 \%$ skim milk, followed by incubation with primary antibodies against $\beta$-actin (1:1,000; cat. no. ab8227; Abcam) and FOXG1 $\left(1: 1,000\right.$; cat. no. ab18987; Abcam) overnight at $4^{\circ} \mathrm{C}$. The membrane was then incubated with a biotin-conjugated goat anti-rabbit IgG (1:5,000; cat. no. BA1003; Wuhan Boster Biological Technology, Ltd.) at room temperature for $1 \mathrm{~h}$. Protein bands were visualized using an enhanced chemiluminescence kit (EMD Millipore) and $\beta$-actin was considered as the internal control.

Reverse transcription-quantitative ( $R T-q) P C R$ ). Total RNA was extracted from U87 and TG-905 cells using TRIzol ${ }^{\circledR}$ reagent (Invitrogen; Thermo Fisher Scientific, Inc) according to the manufacturer's protocol. For the quantification of miRNA expression, RT and qPCR analysis were performed using the Bulge-Loop ${ }^{\mathrm{TM}}$ miRNA qRT-PCR Starter kit (Guangzhou RiboBio Co., Ltd) at $42^{\circ} \mathrm{C}$ for $60 \mathrm{~min}$ and $70^{\circ} \mathrm{C}$ for $10 \mathrm{~min}$. For the quantification of FOXG1 expression, mRNA was converted to cDNA using a PrimeScript ${ }^{\mathrm{TM}} \mathrm{RT}$ reagent kit (Takara Bio, Inc.) at $37^{\circ} \mathrm{C}$ for $15 \mathrm{~min}$ and $85^{\circ} \mathrm{C}$ for $5 \mathrm{sec}$. qPCR was performed using SYBR ${ }^{\circledR}$ Premix Ex Taq ${ }^{\mathrm{TM}}$ (Takara Bio, Inc.). The thermocycling conditions were as follows: Initial denaturation at $95^{\circ} \mathrm{C}$ for $3 \mathrm{~min} ; 40$ cycles of denaturation at $95^{\circ} \mathrm{C}$ for $5 \mathrm{sec}$, annealing at $60^{\circ} \mathrm{C}$ for $30 \mathrm{sec}$ and elongation at $72^{\circ} \mathrm{C}$ for $30 \mathrm{sec}$; and a final extension at $72^{\circ} \mathrm{C}$ for $30 \mathrm{sec}$. The relative expressions levels of FOXG1 and miR-9-3p were calculated using the $2^{-\Delta \Delta \mathrm{Cq}}$ method and normalized to the housekeeping gene $\beta$-actin and U6 rRNA (17). The specific primer sequences were as follows: FOXG1 forward, 5'-GGC TCACGCTCAACGGCATCTACGA-3' and reverse, 5'-GCG GCACCTTCACGAAGCACTTGTT-3'; and $\beta$-actin forward, 5'-GAAGATCAAGATCATTGCTCCT-3' and reverse, 5'-TAC TCCTGCTTGCTGATCCA-3'. The catalog number of miR-9-3p primer is MQPS0002283-1-100, and the catalog number of U6 is MQPS0000002-1-100.

Cell proliferation assay. The growth curves of U87 and TG-905 cells were determined using Cell Counting Kit-8 (CCK-8; Dojindo Molecular Technologies, Inc.) $24 \mathrm{~h}$ after 
transfection, according to the manufacturer's protocol. Cells (7x10\%3ell) were suspended and seeded in 96-well plates overnight. Following incubation, the original culture medium was removed and $100 \mu \mathrm{l}$ fresh medium mixed with CCK-8 at a ratio of 10:1 was added to each well. The absorbance at $450 \mathrm{~nm}$ was measured using a microplate reader (Thermo Fisher Scientific, Inc.) to generate cell growth curves.

Annexin-V/propidium iodide (PI) double-staining assay. Following transection with miR-9-3p mimic or FOXG1 siRNA for $24 \mathrm{~h}, \mathrm{U} 87$ and TG-905 cells were digested using $0.25 \%$ trypsin and centrifuged at $148 \mathrm{x}$ g for $5 \mathrm{~min}$ at room temperature, prior to collection for apoptosis detection. The collected cells were washed with PBS and digested using trypsin. Subsequently, $1 \times 10^{5}$ cells were resuspended in $100 \mu \mathrm{l}$ binding buffer, $5 \mu \mathrm{l}$ Annexin V-FITC and $5 \mu \mathrm{l}$ PI staining solution (BD Biosciences), followed by incubation at room temperature $\left(20-25^{\circ} \mathrm{C}\right)$ for $15 \mathrm{~min}$, according to the manufacturer's protocol. Cell apoptosis was analyzed using a flow cytometer (BD Biosciences) within $1 \mathrm{~h}$ of treatment and the data were analyzed using FlowJo 10.07 software (FlowJo LLC). Cells that were Annexin V-positive were considered to indicate cells undergoing apoptosis.

Dual luciferase reporter assay. miR-9-3p targets were predicted using bioinformatics software programs, including TargetScan (http://www.targetscan.org/), mirDB (http://mirdb. org/) and DIANA TOOLS (http://diana.imis.athena-innovation.gr). U87 and TG-905 cells (4x10\%/well) were plated in 24 -well plates. When the cultures attained $50 \%$ confluence, cells were co-transfected using the Renilla luciferase pRL-TK plasmid (100 ng/ml; Shanghai GenePharma Co., Ltd.) and a recombinant Firefly luciferase pGL3 reporter containing the 3'-untranslated region (3'-UTR) of human FOXG1 ( $2 \mu \mathrm{g} / \mathrm{ml}$; Shanghai GenePharma Co., Ltd.). This was followed by transfection with the miR-9-3p mimic and NC mimic using Lipofectamine ${ }^{\circledR} 2000$ (Invitrogen; Thermo Fisher Scientific, Inc.), according to the manufacturer's protocol. The cells were then collected and lysed for a luciferase assay $24 \mathrm{~h}$ after transfection using a Dual-Luciferase Reporter assay kit (Promega Corporation). Firefly luciferase activity was normalized to Renilla luciferase activity for each tested well.

Statistical analysis. Statistical analyses were conducted using SPSS 20.0 software (IBM Corp.). Data are presented as the mean \pm standard deviation, and each experiment was performed in triplicate. Differences among multiple groups were compared using one-way analysis of variance and Dunnett's or Tukey's post hoc test, and differences between two groups were compared using Dunnett's t-test. $\mathrm{P}<0.05$ was considered to indicate a statistically significant difference.

\section{Results}

miR-9-3p is downregulated in glioma tissues. miR-9-3p has been reported to be downregulated in gastric carcinoma (18) and colorectal cancer (19). To investigate whether the expression of miR-9-3p is abnormal in glioma, the present study performed RT-qPCR to detect the expression level of miR-9-3p mRNA in glioma and adjacent paracancerous glioma tissues. As exhibited in Fig. 1A, miR-9-3p expression was significantly

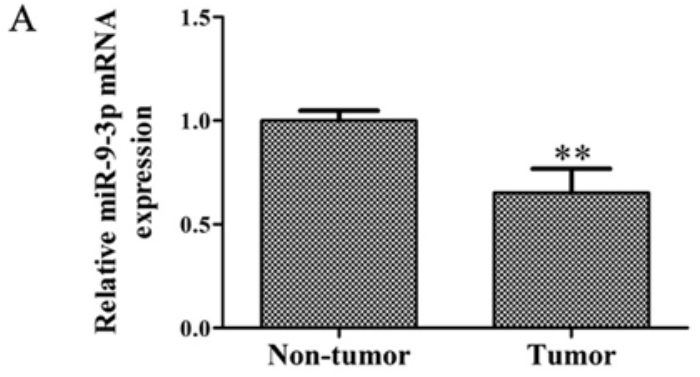

B
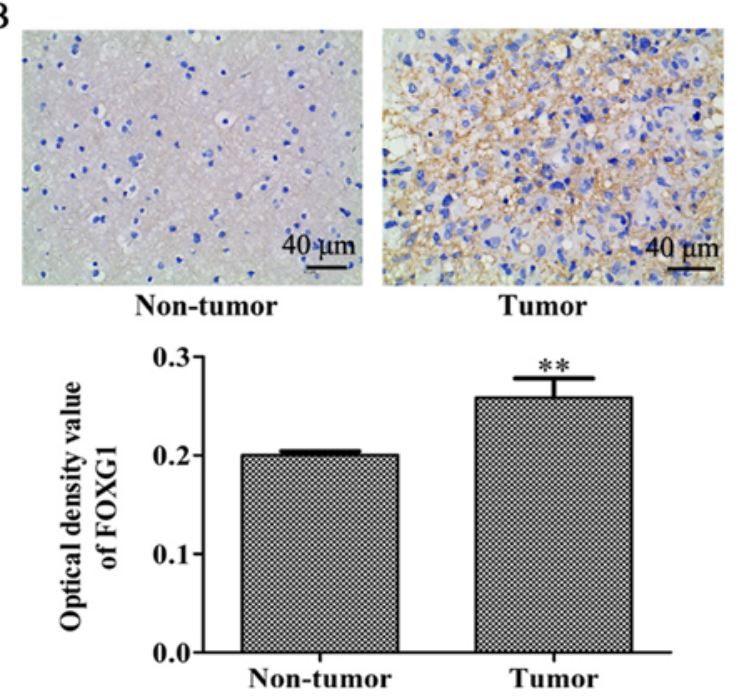

Figure 1. miR-9-3p and FOXG1 are aberrantly expressed in glioma tissues. (A) RT-qPCR was performed to detect the expression level of miR-9-3p in glioma $(n=5)$ and glioma-adjacent tissues $(n=5)$. (B) Immunohistochemistry was performed to detect the expression level of FOXG1 in glioma $(n=5)$ and glioma-adjacent tissues $(n=5){ }^{* *} \mathrm{P}<0.01$ vs. non-tumor group. miR-9-3p, microRNA-9-3p; FOXG1, forkhead box G1; RT-qPCR, reverse transcription-quantitative polymerase chain reaction.

lower in glioma tissues compared with in glioma-adjacent tissues, which indicates a potential role for miR-9-3p in glioma tumorigenesis and progression. To investigate the potential downstream mechanisms of miR-9-3p in glioma, an immunohistochemistry assay was performed to detect the expression level of FOXG1. As presented in Fig. 1B, the protein expression level of FOXG1 in glioma tissue was significantly increased compared with that of glioma-adjacent tissues. In view of the negative regulatory association between miRNA and its target genes, this result reveals that miR-9-3p may have a targeted regulatory association with FOXG1.

Overexpression of miR-9-3p inhibits cell proliferation and increases cell apoptosis. To further investigate the biological functions of miR-9-3p in glioma, U87 MG and TG-905 cells were transfected with miR-9-3p mimic and a NC mimic. RT-qPCR revealed that miR-9-3p expression was significantly increased following transfection with miR-9-3p mimic, compared with the control and NC mimic groups, suggesting high transfection efficiency in U87 MG and TG-905 cells (Fig. 2A and C). CCK-8 and Annexin-V/PI double-staining assays were then performed to determine the proliferation and apoptosis rate in U87 MG and TG-905 cells, and to investigate the influence of miR-9-3p on the progression of glioma. It was revealed that miR-9-3p overexpression significantly suppressed 
A

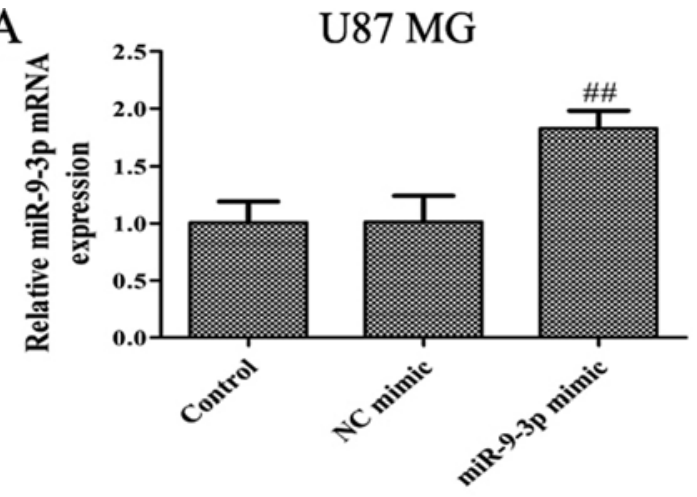

B

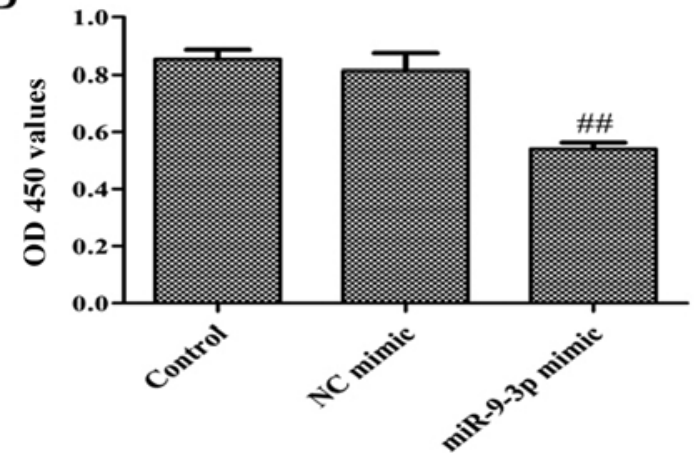

$\mathrm{C}$

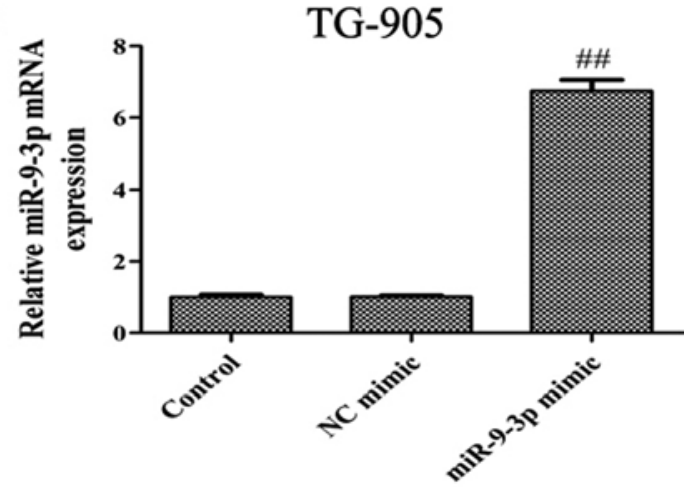

$\mathrm{D}$

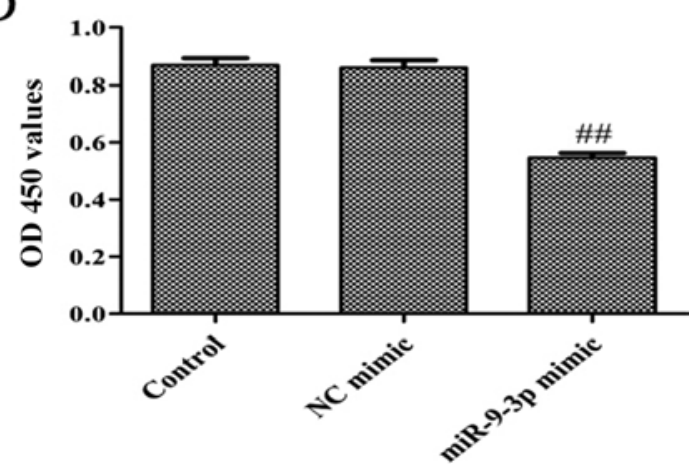

Figure 2. Effects of miR-9-3p overexpression on the proliferation of U87 cells. U87 cells were transfected with miR-9-3p mimic and NC mimic (50 pmol/ml) for $24 \mathrm{~h}$. (A and C) RT-qPCR was applied to quantify the expression level of miR-9-3p in U87 MG and TG-905 cells. (B and D) The cell viability of U87 cells was determined by a CCK-8 assay in U87 MG and TG-905 cells. ${ }^{\#} \mathrm{P}<0.01$ vs. NC mimic group. miR-9-3p, microRNA-9-3p; RT-qPCR, reverse transcription-quantitative polymerase chain reaction; $\mathrm{NC}$, negative control; control, without any treatment.

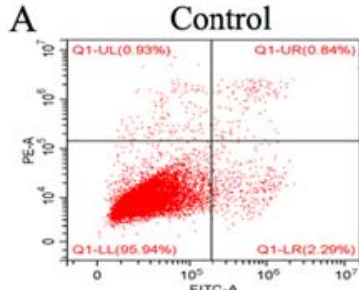

miR-9-3p mimic

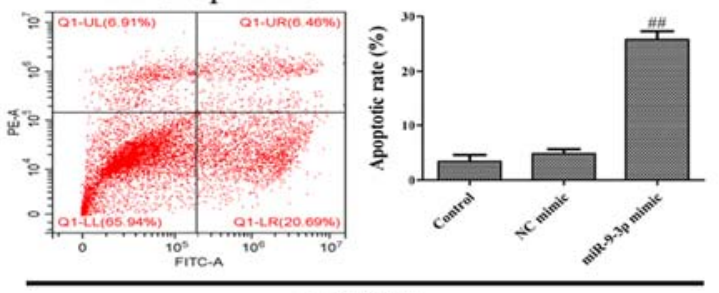

U87 MG

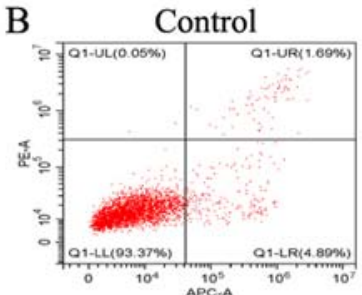

miR-9-3p mimic
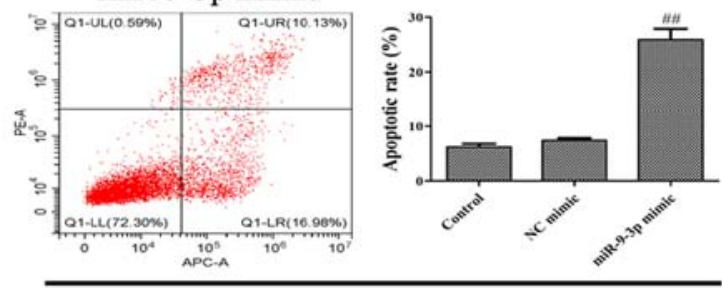

TG-905

Figure 3. Effects of miR-9-3p overexpression on the apoptosis of U87 cells. U87 cells were transfected with miR-9-3p mimic and NC mimic (50 pmol/ml) for $24 \mathrm{~h}$. (A) Annexin-V/PI double-staining assay was applied to detect the cell apoptotic rate in U87 MG cells. (B) Annexin-V/PI double-staining assay was applied to detect the cell apoptotic rate in TG-905 cells. ${ }^{\# \#} \mathrm{P}<0.01$ vs. NC mimic group. miR-9-3p, microRNA-9-3p; NC, negative control; PI, propidium iodide; control, without any treatment.

proliferation (Fig. 2B and D), whilst simultaneously increasing the apoptosis of U87 MG and TG-905 cells (Fig. 3A and B). The present results indicate that miR-9-3p may act as a tumor suppressor and regulate the progression of glioma cells.

FOXG1 is a direct target of miR-9-3p in glioma cells. To determine the mechanism of $\mathrm{miR}-9-3 \mathrm{p}$ regulation of glioma, the present study investigated potential miR-9-3p targets using TargetScan, mirDB and DIANA TOOLS. FOXG1 was selected from several putative miR-9-3p targets as it serves key roles in: Cerebellar development (20), glioma proliferation (21), apoptosis (15) and also predicts prognosis (22). As indicated in Fig. 4A, it was revealed that the 3'-UTR of FOXG1 was contains a conserved putative target site for miR-9-3p. Subsequently, 
$\mathrm{B}$

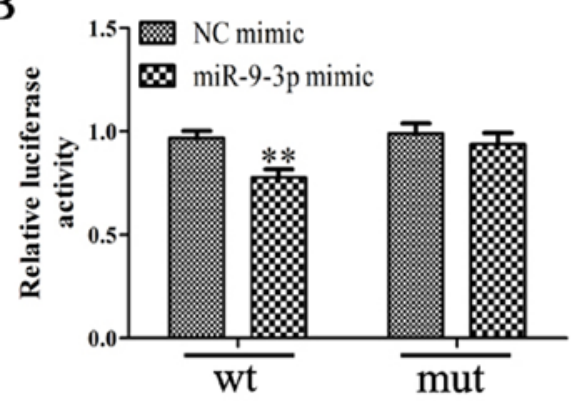

$\mathrm{C}$

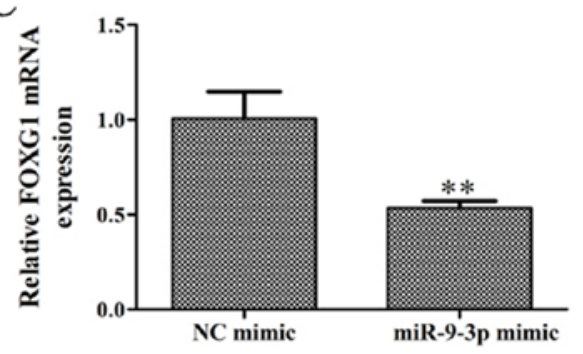

$\mathrm{D}$
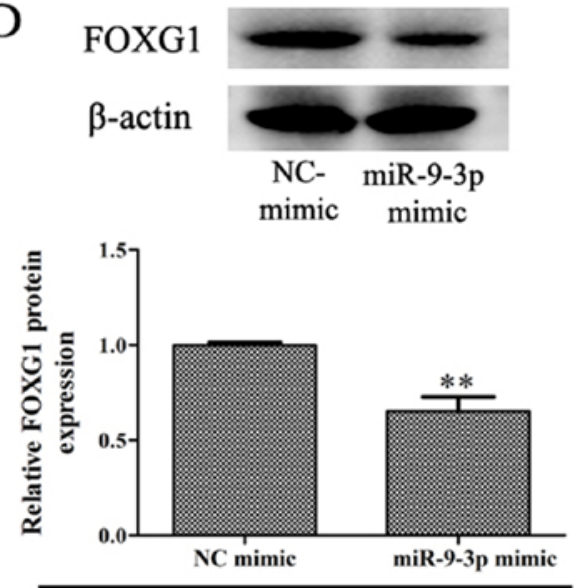

U87 MG
$\mathrm{E}$

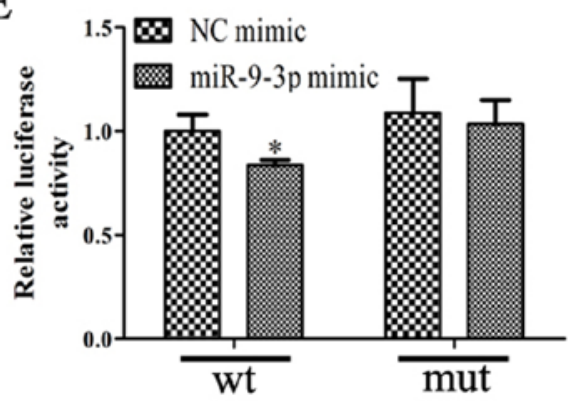

$\mathrm{F}$

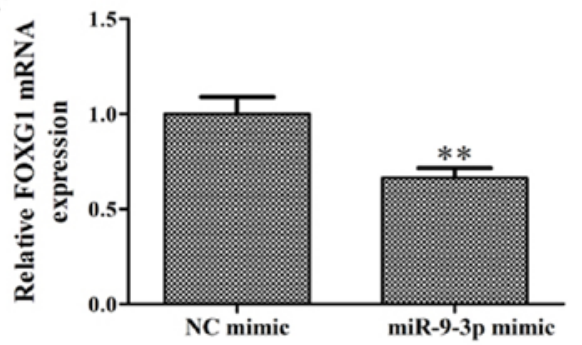

G FOXG1

$\beta$-actin

NC- miR-9-3p

mimic mimic

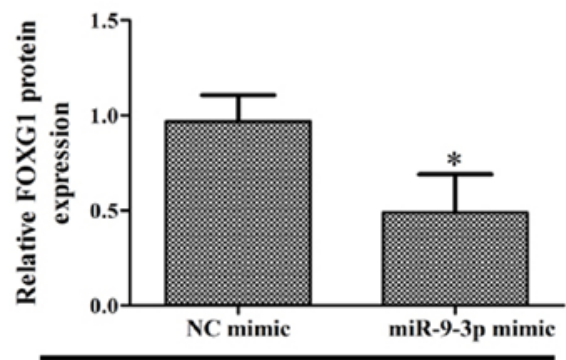

TG-905

Figure 4. FOXG1 is a direct target of miR-9-3p in glioma cells. (A) Bioinformatics analysis of the predicted interactions of miR-9-3p and its binding sites within the 3'-UTR of FOXG1. (B) Relative luciferase activities of FOXG1-wt, and FOXG1-mut were identified using a Dual-Luciferase Reporter assay kit following transfection of U87 MG with miR-9-3p mimic and NC mimic (50 pmol/ml) for $24 \mathrm{~h}$. (C) The expression level of FOXG1 mRNA was examined by RT-qPCR following transfection of U87 MG with miR-9-3p mimic and NC mimic (50 pmol/ml) for $24 \mathrm{~h}$. (D) The expression level of FOXG1 protein was examined by western blot assay following transfection of U87 MG with miR-9-3p mimic and NC mimic (50 pmol/ml) for $48 \mathrm{~h}$. The relative intensity of FOXG1 protein is presented as a bar graph. (E) Relative luciferase activities of FOXG1-wt, and FOXG1-mut were identified using a Dual-Luciferase Reporter assay kit following transfection of TG-905 cells with miR-9-3p mimic and NC mimic (50 pmol/ml) for $24 \mathrm{~h}$. (F) The expression level of FOXG1 mRNA was examined by RT-qPCR following transfection of TG-905 cells with miR-9-3p mimic and NC mimic (50 pmol/ml) for 24 h. (G) The expression level of FOXG1 protein was examined by western blot assay following transfection of TG-905 cells with miR-9-3p mimic and NC mimic (50 pmol/ml) for $48 \mathrm{~h}$. The relative intensity of FOXG1 protein is presented as a bar graph. * $\mathrm{P}<0.05,{ }^{* *} \mathrm{P}<0.01$ vs. NC mimic group. FOXG1, forkhead box G1; miR-9-3p, microRNA-9-3p; 3'-UTR, 3'-untranslated region; wt, wild-type; mut, mutant; NC, negative control.

dual-luciferase assays were performed to determine whether FOXG1 is a direct target of miR-9-3p. The results indicated that overexpression of miR-9-3p suppressed the activity of luciferase reporters containing FOXG1 3'-UTR in U87 MG and TG-905 cells (Fig. 4B and E). In addition, the RT-qPCR assay revealed that miR-9-3p overexpression was significantly inhibited the expression of FOXG1 mRNA in U87 MG and TG-905 cells (Fig. 4C and F). Western blotting analysis revealed that the overexpression of miR-9-3p significantly inhibited the expression of FOXG1 protein (Fig. 4D and G). In summary, the 

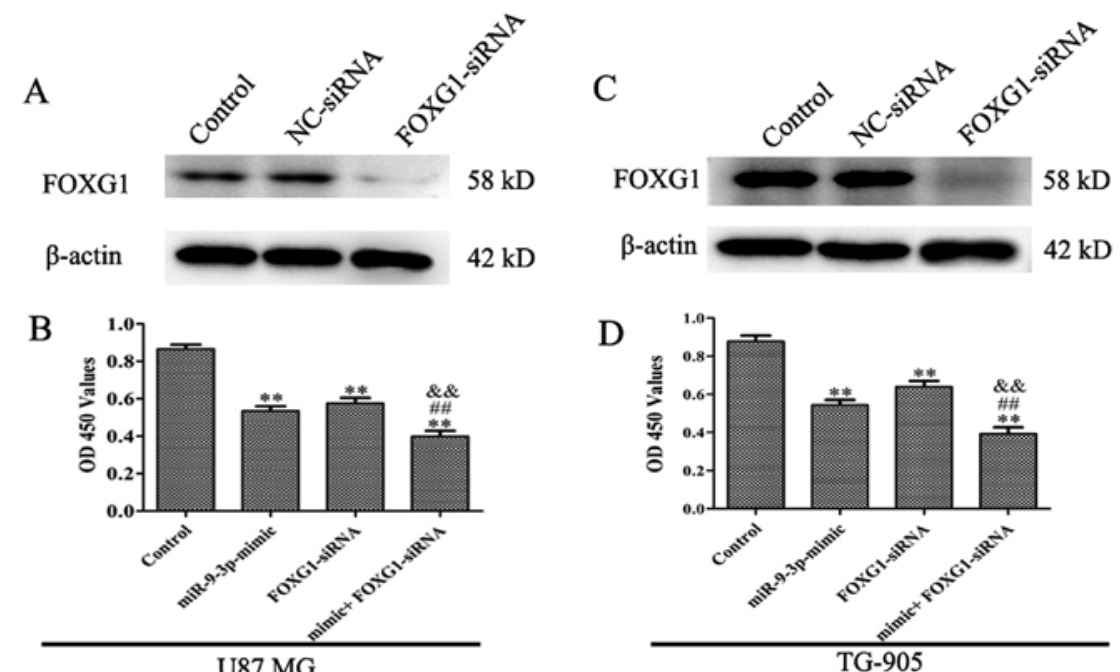

Figure 5. miR-9-3p regulates cell proliferation by inhibiting FOXG1. (A and C) Western blotting was used to detect the protein expression of FOXG1 following transfection of U87 MG and TG-905 cells with FOXG1 siRNA (40 pmol/ml) for $48 \mathrm{~h}$. (B and D) Relative cell viability was determined by a Cell Counting Kit-8 assay following transfection of U87 MG and TG-905 cells with FOXG1 (40 pmol/ml) for $24 \mathrm{~h}$. ${ }^{* *} \mathrm{P}<0.01$ vs. control group; ${ }^{\# /} \mathrm{P}<0.01 \mathrm{vs}$. miR-9-3p mimic group; \&\&P<0.01 vs. FOXG1-siRNA group. FOXG1, forkhead box G1; miR-9-3p, microRNA-9-3p; siRNA, small interfering RNA; control, without any treatment.
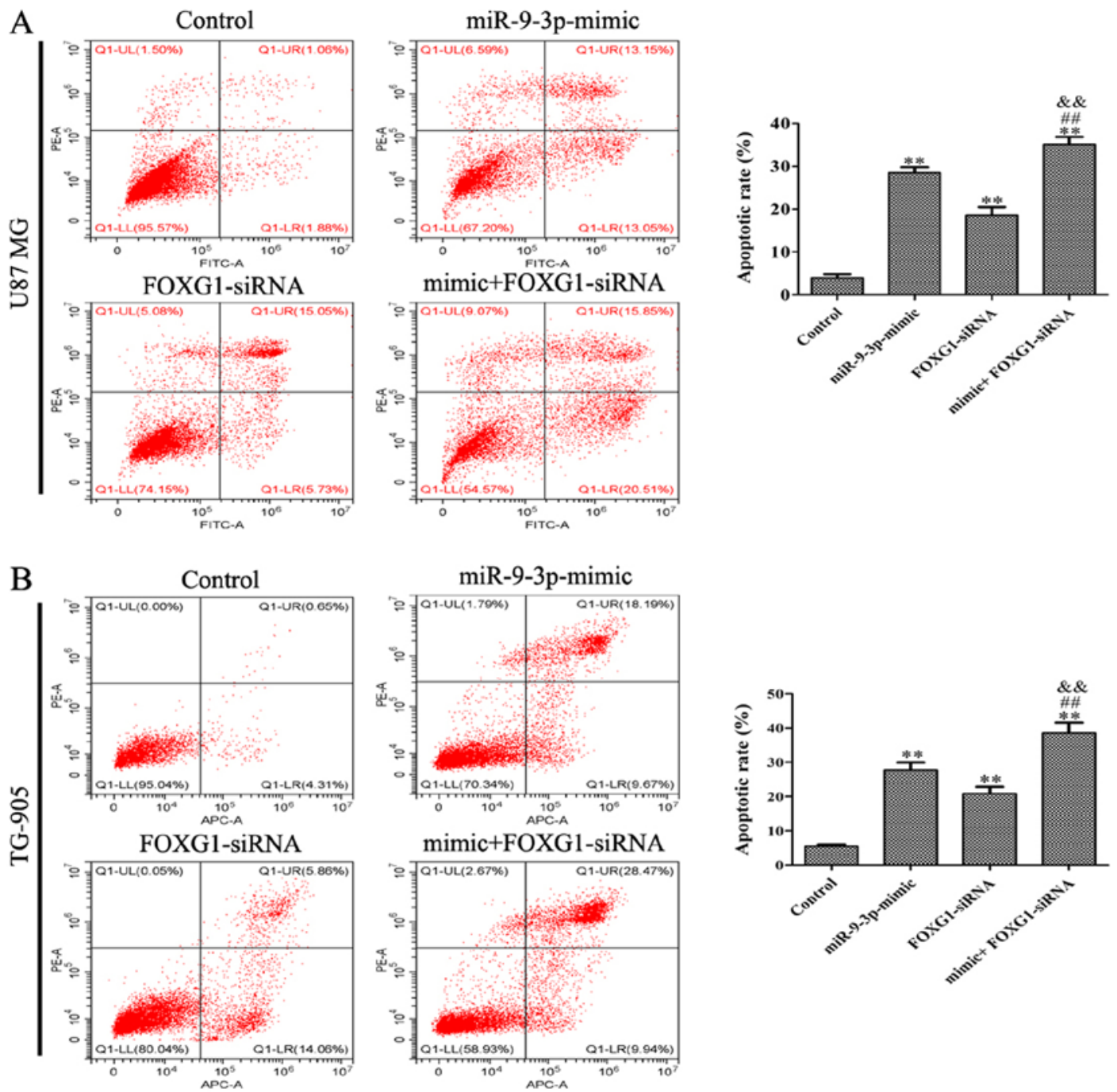

Figure 6. miR-9-3p regulates cell apoptosis by inhibiting FOXG1. (A) Annexin-V/PI double-staining assay was performed to detect the cell apoptotic rate of U87 MG cells following transfection with FOXG1 siRNA (40 pmol/ml) for $24 \mathrm{~h}$. (B) Annexin-V/PI double-staining assay was applied to detect the cell apoptotic rate of TG-905 cells following transfection with FOXG1 siRNA ( $40 \mathrm{pmol} / \mathrm{ml})$ for $24 \mathrm{~h}$. ${ }^{* *} \mathrm{P}<0.01$ vs. control group; ${ }^{* \#} \mathrm{P}<0.01 \mathrm{vs} \mathrm{miR}-9-3 \mathrm{p}$ mimic group; \&\&P<0.01 vs. FOXG1-siRNA group. miR-9-3p, microRNA-9-3p; FOXG1, forkhead box G1; PI, propidium iodide; siRNA, small interfering RNA; control, without any treatment. 
present study demonstrated that miR-9-3p targets FOXG1 and suppresses its expression in glioma cells.

FOXG1 gene silencing enhances the effect of miR-9-3p mimic on cell proliferation inhibition and apoptosis. To determine whether FOXG1 influences the effect of miR-9-3p on the proliferation and apoptosis of U87 cells, siRNA was used to downregulate FOXG1 expression. As revealed in Fig. 5A and C, FOXG1 protein expression was downregulated following transfection with FOXG1 siRNA, suggesting high transfection efficiency of FOXG1 siRNA in U87 MG and TG-905 cells. CCK-8 and flow cytometry results revealed that siFOXG1 significantly decrease the proliferation and increased apoptosis in U87 cells. Furthermore, silencing of FOXG1 enhanced the effect of the miR-9-3p mimic on cell proliferation (Fig. 5B and D) and apoptosis (Fig. 6A and B). The present results demonstrated that miR-9-3p regulates cell proliferation and apoptosis via the inhibition of FOXG1.

\section{Discussion}

Glioma is the most common type of brain tumor, with an incidence of 6 per 100,000 annually, worldwide (23). Numerous studies have focused on the effects of miRNA dysregulation in various types of human cancer. miRNAs have been revealed to influence a variety of biological processes by negatively regulating the protein levels of target onco- and tumor suppressor genes; therefore, miRNAs themselves serve as oncogenes or tumor suppressor genes in various types of human tumors $(24,25)$. A number of studies have revealed the aberrant regulation of miR-9 in multiple tumor types. For example, upregulation of miR-9 was shown to promote tumor metastasis via the targeting E-cadherin in serous ovarian cancer (26). Moreover, Tang et al (27) reported that miR-9 functions as a tumor suppressor in ovarian serous carcinoma, as it inhibits cell proliferation, migration and invasion via regulation of talin 1. By contrast, Zhu et al (28) demonstrated that miR-9 serves an oncogenic role by regulating the proliferation of osteosarcoma cells via direct targeting of GCIP. Additionally, miR-9 has been reported to be upregulated in certain glioma specimens and cells, and may significantly promote tumorigenesis and angiogenesis (11). However, Yang et al (12) reported that miR-9-3p is downregulated in high-grade gliomas compared with non-tumor tissues, and the overexpression of miR-9-3p increased apoptosis of glioma cells. Therefore, further investigation is required to identify the expression patterns and mechanisms of action of miR-9, in relation to glioma. The present study revealed that the expression level of miR-9-3p is significantly lower in glioma tissues compared with glioma-adjacent tissues.

The present study is not without limitations. For example, paraffin-embedded in situ hybridization was unable to be performed, which may have been useful in determining direct miR-9 expression. Prospective studies will focus on determining the difference between tumor and adjacent normal tissue samples collected from the same patient, in order to accurately elucidate the differential expression of miR-9.

Overexpression of miR-9 has been revealed to inhibit cell proliferation and invasion in pancreatic cancer (29), hepatocellular carcinoma (30) and nasopharyngeal carcinoma (31). The present study discovered that an overexpression of miR-9-3p markedly inhibited the proliferation, and increased the apoptosis, of U87 cells and the current results demonstrated that miR-9-3p may act as a tumor suppressor, and its overexpression may suppress glioma development, potentially improving patient prognosis.

As previously described, miR-9 has numerous targets; it can suppress the gene expression of StAR related lipid transfer domain containing 13 (32), BLCAP apoptosis inducing factor (33), SRY-box transcription factor 7 (34) and ATP binding cassette subfamily B member 1 (35), by serving either an oncogenic role or acting as a tumor suppressor gene. The FOXG1 gene is located in the q12 region of chromosome 14 and the protein it encodes contains 489 amino acids, is an important transcription factor and serves an important role in the regulation of telencephalic development, neuronal differentiation and neurogenesis $(36,37)$. Studies have identified that FOXG1 serves a role similar to that of oncogenes in the occurrence and development of certain tumors types, including medulloblastoma (38), non-small cell lung cancer (39) and ovarian cancer (40). In addition, a low expression of FOXG1 serves as an important indicator of good prognosis in patients with glioma (22). Bredenkamp et al (41) demonstrated that the FOXG1 3'UTR has a conserved recognition site specific to miR-9, in mammals. However, to the best of our knowledge, whether miR-9 can regulate the progression of glioma via targeting FOXG1 has not been reported. The present study identified that the 3'-UTR of FOXG1 contained a conserved putative target site specific to miR-9-3p in U87 cells, and a dual-luciferase reporter assay revealed that miR-9-3p overexpression suppressed luciferase activity. Furthermore, it was identified that miR-9-3p overexpression inhibited the mRNA and protein expression levels of FOXG1. The present results suggest that FOXG1 is a direct functional target of miR-9-3p. Additionally, the present study demonstrated that the inhibition of FOXG1 not only inhibited cell proliferation and increased cell apoptosis, but also enhanced the effect of miR-9-3p mimic on the proliferation and apoptosis of U87 MG and TG-905 cells, indicating that miR-9-3p regulates cell proliferation and apoptosis via targeting FOXG1 in glioma.

In summary, the present study demonstrated that miR-9-3p functions as a tumor suppressor in glioma. It was identified that miR-9-3p inhibits glioma progression via targeting FOXG1. Although miRNA-based therapeutics are still under development, the present results are support the hypothesis that miR-9-3p and FOXG1 may represent novel and effective therapeutic targets for glioma treatment in the future.

\section{Acknowledgements}

Not applicable.

\section{Funding}

The present study was funded by the National Natural Science Foundation of China (grant no. 81560732).

\section{Availability of data and materials}

The datasets used or analyzed during the present study are available from the corresponding author upon reasonable request. 


\section{Authors' contributions}

JZ and XT conceived and designed the present study. JZ, HZ and HD performed the experiments and analyzed the data. JZ drafted the initial manuscript. XT critically revised the manuscript for important intellectual content and supervised the study. All authors have read and approved the final version of this manuscript.

\section{Ethics approval and consent to participate}

The present study was approved by the Ethics Review Board of the Affiliated Hospital of Xizang Minzu University (Xianyang, China), and written informed consent was obtained from all patients prior to the study start (approval number: 201505).

\section{Patient consent for publication}

Not applicable.

\section{Competing interests}

The authors declare that they have no competing interests.

\section{References}

1. Di Stefano AL, Enciso-Mora V, Marie Y, Desestret V, Labussière M, Boisselier B, Mokhtari K, Idbaih A, Hoang-Xuan K, Delattre JY, et al: Association between glioma susceptibility loci and tumour pathology defines specific molecular etiologies. Neuro Oncol 15: 542-547, 2013.

2. Li G, Shen J, Cao J, Zhou G, Lei T, Sun Y, Gao H, Ding Y, $\mathrm{Xu} \mathrm{W}$, Zhan Z, et al: Alternative splicing of human telomerase reverse transcriptase in gliomas and its modulation mediated by CX-5461. J Exp Clin Cancer Res 37: 78, 2018.

3. Parker RG, Janjan NA and Selch MT: Malignant tumors of the central nervous system. In: Radiation Oncology for Cure and Palliation. Springer, Berlin, Heidelberg, 2003.

4. Fuller GN and Scheithauer BW: The 2007 revised world health organization (WHO) classification of tumours of the central nervous system: Newly codified entities. Brain Pathol 17: 304-307, 2010

5. Theeler BJ and Groves MD: High-grade gliomas. Curr Treat Options Neurol 13: 386-399, 2011.

6. Ke C, Tran K, Chen Y, Di Donato AT, Yu L, Hu Y, Linskey ME, Wang PH, Limoli CL and Zhou YH: Linking differential radiation responses to glioma heterogeneity. Oncotarget 5: 1657-1665, 2014.

7. Fischer U, Struss AK, Hemmer D, Pallasch CP, Steudel WI and Meese E: Glioma-expressed antigen 2 (GLEA2): A novel protein that can elicit immune responses in glioblastoma patients and some controls. Clin Exp Immunol 126: 206-213, 2010.

8. Kwok HH, Poon PY, Mak KH, Zhang LY, Liu P, Zhang H, Mak NK, Yue PY and Wong RN: Role of G3BP1 in glucocorticoid receptor-mediated microRNA-15b and microRNA-23a biogenesis in endothelial cells. Cell Mol Life Sci 74: 3613-3630, 2017.

9. Xiong W, Ran J, Jiang R, Guo P, Shi X, Li H, Lv X, Li J and Chen D: MIRNA-320a inhibits glioma cell invasion and migration by directly targeting aquaporin 4. Oncol Rep 39: 1939-1947, 2018.

10. Liu DK, Wei YJ, Guo Y, Wang J and Wang GH: MiRNA-93 functions as an oncogene in glioma by directly targeting RBL2. Eur Rev Med Pharmacol Sci 22: 2343-2350, 2018.

11. Chen X, Yang F, Zhang T, Wang W, Xi W, Li Y, Zhang D, Huo Y, Zhang J, Yang A and Wang T: MiR-9 promotes tumorigenesis and angiogenesis and is activated by MYC and OCT4 in human glioma. J Exp Clin Cancer Res 38: 99, 2019.

12. Yang L, Mu Y, Cui H, Liang Y and Su X: MiR-9-3p augments apoptosis induced by $\mathrm{H} 2 \mathrm{O} 2$ through down regulation of Herpud1 in glioma. PLoS One 12: e0174839, 2017.

13. Golson ML and Kaestner KH: Fox transcription factors: From development to disease. Development 143: 4558-4570, 2016.
14. Creagh EM, Conroy $\mathrm{H}$ and Martin SJ: Caspase-activation pathways in apoptosis and immunity. Immunol Rev 193: 10-21, 2003.

15. Chen J, Wu X, Xing Z, Ma C, Xiong W, Zhu X and He X: FOXG1 Expression is elevated in glioma and inhibits glioma cell apoptosis. J Cancer 9: 778-783, 2018.

16. Shibata M, Kurokawa D, Nakao H, Ohmura T and Aizawa S: MicroRNA-9 modulates Cajal-Retzius cell differentiation by suppressing Foxg1 expression in mouse medial pallium. J Neurosci 28: 10415-10421, 2008.

17. Livak KJ and Schmittgen TD: Analysis of relative gene expression data using real-time quantitative PCR and the 2(-Delta Delta C(T)) method. Methods 25: 402-408, 2001.

18. Luo H, Zhang H, Zhang Z, Zhang X, Ning B, Guo J, Nie N, Liu B and Wu X: Down-regulated miR-9 and miR-433 in human gastric carcinoma. J Exp Clin Cancer Res 28: 82, 2009.

19. Zhu M, Xu Y, Ge M, Gui Z and Yan F: Regulation of UHRF1 by microRNA-9 modulates colorectal cancer cell proliferation and apoptosis. Cancer Sci 106: 833-839, 2015.

20. Kersigo J, D'Angelo A, Gray BD, Soukup GA and Fritzsch B: The role of sensory organs and the forebrain for the development of the craniofacial shape as revealed by Foxg1-cre-mediated microRNA loss. Genesis 49: 326-341, 2011.

21. Wang L, Wang J, Jin T, Zhou Y and Chen Q: FoxG1 facilitates proliferation and inhibits differentiation by downregulating FoxO/Smad signaling in glioblastoma. Biochem Biophys Res Commun 504: 46-53, 2018.

22. Schäfer S, Behling F, Skardelly M, Koch M, Ott I, Paulsen F, Tabatabai G and Schittenhelm J: Low FoxG1 and high Olig-2 labelling indices define a prognostically favourable subset in isocitrate dehydrogenase (IDH)-mutant gliomas. Neuro Appl Neurobiol 44: 207-223, 2018.

23. Goldbrunner R, Ruge M, Kocher M, Lucas CW, Galldiks N and Grau S: The treatment of gliomas in adulthood. Dtsch Arztebl Int 115: 20-21, 2018.

24. Wei Y, Schober A and Weber C: Pathogenic arterial remodeling: The good and bad of microRNAs. Am J Physiol Heart Circu Physiol 304: H1050-H1059, 2013.

25. Li B, Liu YH, Sun AG, Huan LC, Li HD and Liu DM: MiR-130b functions as a tumor promoter in glioma via regulation of ERK/MAPK pathway. Eur Rev Med Pharmacol Sci 21: 2840-2846, 2017

26. Zhou B, Xu H, Xia M, Sun C, Li N, Guo E, Guo L, Shan W, Lu H, Wu Y, et al: Overexpressed miR-9 promotes tumor metastasis via targeting E-cadherin in serous ovarian cancer. Front Med 11: 214-222, 2017.

27. Tang H, Yao L, Tao X, Yu Y, Chen M, Zhang R and Xu C: Mir-9 functions as a tumor suppressor in ovarian serous carcinoma by targeting tln1. Int J Mol Med 32: 381-388, 2013.

28. Zhu SW, Li JP, Ma XL, Ma JX, Yang Y, Chen Y and Liu W: MiR-9 modulates osteosarcoma cell growth by targeting the GCIP tumor suppressor. Asian Pac J Cancer Prev 16: 4509-4513, 2015.

29. Wang J, Wang B, Ren H and Chen W: Mir-9-5p inhibits pancreatic cancer cell proliferation, invasion and glutamine metabolism by targeting GOT1. Biochem Biophy Res Commun 509: 241-248, 2019.

30. Han Y, Liu Y, Fu X, Zhang Q, Huang H, Zhang C, Li W and Zhang J: MiR-9 inhibits the metastatic ability of hepatocellular carcinoma via targeting beta galactoside alpha-2,6-sialyltransferase 1. J Physiol Biochem 74: 491-501, 2018.

31. Ding Y, Pan Y, Liu S, Jiang F and Jiao J: Elevation of MiR-9-3p suppresses the epithelial-mesenchymal transition of nasopharyngeal carcinoma cells via down-regulating FN1, ITGB1 and ITGAV. Cancer Biol Ther 18: 414-424, 2017.

32. Chen L, Hu W, Li G, Guo Y, Wan Z and Yu J: Inhibition of miR-9-5p suppresses prostate cancer progress by targeting StarD13. Cell Mol Biol Lett 24: 20, 2019.

33. Chen Y, Zhang S, Zhao R, Zhao Q and Zhang T: Upregulated miR-9-3p promotes cell growth and inhibits apoptosis in medullary thyroid carcinoma by targeting BLCAP. Oncol Res 25: 1215-1222, 2017.

34. Han L, Wang W, Ding W and Zhang L: MiR-9 is involved in TGF- $\beta 1$-induced lung cancer cell invasion and adhesion by targeting SOX7. J Cell Mol Med 21: 2000-2008, 2017.

35. Li Y, Zhao L, Li N, Miao Y, Zhou H and Jia L: MiR-9 regulates the multidrug resistance of chronic myelogenous leukemia by targeting ABCB1. Oncol Rep 37: 2193-2200, 2017.

36. Manuel MN, Martynoga B, Molinek MD, Quinn JC, Kroemmer C, Mason JO and Price DJ: The transcription factor Foxg1 regulates telencephalic progenitor proliferation cell autonomously, in part by controlling Pax6 expression levels. Neural Dev 6: 9, 2011. 
37. Brancaccio M, Pivetta C, Granzotto M, Filippis $\mathrm{C}$ and Mallamaci A: Emx2 and Foxg1 inhibit gliogenesis and promote neuronogenesis. Stem Cells 28: 1206-1218, 2010.

38. Adesina AM, Nguyen Y, Mehta V, Takei H, Stangeby P, Crabtree S, Chintagumpala M and Gumerlock MK: FOXG1 dysregulation is a frequent event in medulloblastoma. J Neurooncol 85: 111-122, 2007.

39. Ji KX, Cui F, Qu D, Sun RY, Sun P, Chen FY, Wang SL and Sun HS: MiR-378 promotes the cell proliferation of non-smal cell lung cancer by inhibiting FOXG1. Eur Rev Med Pharmacol Sci 22: 1011-1019, 2018
40. Chan DW, Liu VW, To RM, Chiu PM, Lee WY, Yao KM, Cheung AN and Ngan HY: Overexpression of FOXG1 contributes to TGF- $\beta$ resistance through inhibition of p21WAF1/CIP1expression in ovarian cancer. Br J Cancer 101: 1433-1443, 2009.

41. Bredenkamp N, Seoighe C and Illing N: Comparative evolutionary analysis of the FoxG1 transcription factor from diverse vertebrates identifies conserved recognition sites for microRNA regulation. Dev Genes Evol 217: 227-233, 2007. 